\title{
A HOMOGENIZATION TECHNIQUE FOR ELASTO-PLASTIC COMPOSITES
}

\author{
F. Covezzi ${ }^{1}$, S. de Miranda ${ }^{1}$, S. Marfia ${ }^{2}$, and E. Sacco ${ }^{2}$ \\ ${ }^{1}$ DICAM, University of Bologna \\ Viale del Risorgimento, Bologna, Italy \\ e-mail: \{federica.covezzi3,stefano.demiranda\}@unibo.it \\ 2 DICeM, University of Cassino and Southern Lazio \\ Via G. di Biasio 43, Cassino, Italy \\ e-mail: \{marfia,sacco\}@unicas.it
}

Keywords: Composite materials, homogenization, nonuniform TFA, complementary formulation, hardening.

\begin{abstract}
The present study proposes a homogenization technique for the estimation of the overall behavior of composite materials characterized by a nonlinear behavior. A mixed nonuniform TFA procedure is proposed in order to study the mechanical response of periodic composites characterized by linear isotropic hardening plasticity. As the case of periodic composites is considered, the homogenization is performed on a repetitive unit cell that plays the equivalent role of representative volume element for random media. A numerical application is performed to test the effectiveness of the proposed technique. In particular, the homogenization results are compared with the ones carried out by micromechanical nonlinear finite element analyses.
\end{abstract}




\section{INTRODUCTION}

The popularity and the use of composite materials have recently increased in the realm of structural engineering, due to their high performances deriving from their superior properties in terms of light weight and resistance. For this reason, effective tools able to predict the mechanical behavior of these media at different scales are required in the design of composite materials. A significant contribution is given by homogenization techniques, which allow to determine the overall behavior of a composite medium starting from its microscopic properties and finally to derive an equivalent homogenized material for numerical computations.

Among the homogenization techniques, an interesting approach for the investigation of the nonlinear response of composites is the Transformation Field Analysis (TFA), originally proposed in [1]. This technique takes into account the nonlinear behavior of the constituents, introducing an approximation of the inelastic strain. In the literature, several TFA schemes have been developed, differing mainly in the assumption concerning the distribution of the inelastic strain and in the computation of the internal variable evolution $[2,3,4,5,6]$.

Recently, a new mixed nonuniform TFA (MxTFA) scheme has been presented by the authors [7], with the aim to improve the description of the inelastic strain field and the computation of the evolutive problem. In this paper, an extension of the MxTFA to the analysis of periodic composites characterized by linear isotropic hardening plasticity is presented. In particular, a mixed variational approach, originally proposed in [8] and recently implemented in $[9,10]$, involving the weak form of compatibility and plastic admissibility equations is adopted to derive the evolution laws of the internal variables in the TFA framework. In fact, the literature shows how stress recovery techniques are able to produce very good results [11, 12].

According to the MxTFA, the RVE is subdivided into subsets and in each subset a uniform plastic multiplier and equilibrated, linearly varying stresses are assumed. As a result, the inelastic strain distribution depends on the assumed distributions of stresses and plastic multiplier, that are the independent variables of the problem, and whose parameters are simultaneously evaluated by enforcing the compatibility condition and the elasto-plastic consistency condition.

The effectiveness of the proposed procedure is shown through a numerical example in the framework of 2D plane stress hardening plasticity, where the results are compared with the ones carried out by micromechanical nonlinear finite element analyses.

In the following, the Voigt notation is adopted; the strain and stress tensors are reported as the vectors with 3 components $\boldsymbol{\varepsilon}=\left\{\begin{array}{lll}\varepsilon_{11} & \varepsilon_{22} & \gamma_{12}\end{array}\right\}^{T}$ and $\boldsymbol{\sigma}=\left\{\begin{array}{ll}\sigma_{11} & \sigma_{22} \\ \sigma_{12}\end{array}\right\}^{T}$, respectively.

\section{MxFTA HOMOGENIZATION WITH HARDENING PLASTICITY}

Let a composite material be considered assuming a periodic microstructure, so that the micromechanical analysis and the homogenization of the material can be performed on a unit cell (UC) .

The micromechanical and homogenization problem for the UC denoted by $\Omega$ consists in computing the periodic displacement field $\widetilde{\mathbf{u}}$, the strain field $\varepsilon$, the periodic strain field $\widetilde{\varepsilon}$, the inelastic strain field $\boldsymbol{\pi}=\left\{\pi_{11} \pi_{22} \pi_{12}\right\}^{T}$, the stress field $\boldsymbol{\sigma}$ and its average value $\overline{\boldsymbol{\sigma}}$, for a prescribed value of the average strain $\bar{\varepsilon}$ assigned in $\Omega$. To this end the compatibility, the constitutive, the equilibrium with zero body forces equations, with periodic suitable boundary conditions as well as the strain and the stress average equations have to be satisfied.

The total strain at the point $\mathrm{x}$ of the UC depends on the contributions deriving from the average strain $\bar{\varepsilon}$ and the inelastic strain $\pi$. The periodic strain field $\widetilde{\varepsilon}$ can be therefore split into: 


$$
\widetilde{\varepsilon}=\widetilde{\varepsilon}^{\varepsilon}+\widetilde{\varepsilon}^{\pi} \quad \text { with } \widetilde{\varepsilon}^{\varepsilon}=\mathbf{L}^{\varepsilon} \bar{\varepsilon}, \widetilde{\varepsilon}^{\pi}=\mathbf{L}^{\pi} \boldsymbol{\pi}
$$

with $\widetilde{\varepsilon}^{\varepsilon}$ and $\widetilde{\varepsilon}^{\pi}$ the perturbation strains due to the average strain $\bar{\varepsilon}$ and to the presence of the inelastic field $\boldsymbol{\pi}$, respectively; $\mathbf{L}^{\varepsilon}$ and $\mathbf{L}^{\pi}$ are localization matrices depending on the point $\mathbf{x}$.

Assuming an associative evolution law, the evolution of the inelastic strain is governed by:

$$
\dot{\boldsymbol{\pi}}=\dot{\gamma} \mathbf{N}(\boldsymbol{\sigma}) \quad \text { with } \quad \mathbf{N}(\boldsymbol{\sigma})=\frac{\partial f}{\partial \boldsymbol{\sigma}},
$$

where $\gamma$ is the plastic multiplier and $f$ is the yield function, satisfying the Kuhn-Tucker and consistency conditions:

$$
f(\boldsymbol{\sigma}) \leq 0, \quad \dot{\gamma} \geq 0, \quad f(\boldsymbol{\sigma}) \dot{\gamma}=0, \quad \dot{f}(\boldsymbol{\sigma}) \dot{\gamma}=0 .
$$

The compatibility condition and the Kuhn-Tucker conditions (3) can be rewritten in the weak form as:

$$
\begin{aligned}
& \int_{\Omega} \delta \boldsymbol{\sigma}^{T} \mathbf{C}^{-1} \boldsymbol{\sigma} d V-\int_{\Omega} \delta \boldsymbol{\sigma}^{T}(\overline{\boldsymbol{\varepsilon}}+\mathbf{D} \widetilde{\mathbf{u}}-\boldsymbol{\pi}) d V=0 \quad \forall \delta \boldsymbol{\sigma}: \mathbf{D}^{T} \delta \boldsymbol{\sigma}=\mathbf{0}, \\
& \int_{\Omega} \delta \gamma f(\boldsymbol{\sigma}) d V \leq 0, \quad \dot{\gamma} \geq 0, \quad \int_{\Omega} \delta \gamma f(\boldsymbol{\sigma}) \dot{\gamma} d V=0 \quad \forall \delta \gamma \geq 0,
\end{aligned}
$$

where $\mathbf{D}$ is the compatibility matrix operator, $\mathbf{D}^{T}$ the equilibrium operator, $\mathbf{C}$ the material constitutive matrix and $V$ the volume of $\Omega$.

Considering plane stress elasto-plasticity with a von Mises yield criterion in the framework of linear isotropic hardening, the limit function is:

$$
f(\boldsymbol{\sigma})=\frac{3}{2} \boldsymbol{\sigma}^{T} \mathbf{M} \boldsymbol{\sigma}-\left(\sigma_{y}+H \bar{\varepsilon}_{p}\right)^{2},
$$

where

$$
\mathbf{M}=\frac{1}{3}\left[\begin{array}{rrr}
2 & -1 & 0 \\
-1 & 2 & 0 \\
0 & 0 & 6
\end{array}\right]
$$

$\sigma_{y}$ is the flow stress, $H$ is the hardening parameter, and $\bar{\varepsilon}_{p}$ is the accumulated plastic strain, whose evolution is defined as:

$$
\dot{\bar{\varepsilon}}_{p}=\sqrt{\frac{2}{3}}\|\dot{\boldsymbol{\pi}}\|
$$

Let the UC be divided into $n$ subsets $\Omega^{j}$ with $j=1, . ., n$, each of them characterized by a volume $V^{j}$, such that:

$$
\Omega=\bigcup_{j=1}^{n} \Omega^{j}, \quad V=\sum_{j=1}^{n} V^{j} .
$$

A representation form is assumed for both the stress and plastic multiplier fields. In particular, it is assumed that on the $j$-th subset $\Omega^{j}$ :

$$
\boldsymbol{\sigma}^{j}=\mathbf{P}^{j} \widehat{\boldsymbol{\sigma}}^{j}
$$




$$
\dot{\gamma}^{j}=\sum_{i=1}^{\rho} r_{i}(\mathbf{x}) \dot{\widehat{\gamma}}_{i}^{j}=\left(\mathbf{r}^{j}\right)^{T} \dot{\hat{\gamma}}^{j},
$$

where $\mathbf{P}^{j}$ is a $3 \times p$ matrix collecting a set of $p$ self-equilibrated stress modes, $\widehat{\boldsymbol{\sigma}}^{j}$ the vector of the unknown $p$ stress parameters, $\mathbf{r}^{j}$ and $\dot{\widehat{\gamma}}^{j}$ are the two vectors collecting the $\rho$ approximation functions and coefficients of a linear combination, respectively. In particular, it is assumed a linear approximation for the stress field in the subset $\Omega^{j}$; consequently, it results $p=7$ and

$$
\mathbf{P}^{j}=\left[\begin{array}{rrrrrrr}
1 & 0 & 0 & x_{2} & 0 & x_{1} & 0 \\
0 & 1 & 0 & 0 & x_{1} & 0 & x_{2} \\
0 & 0 & 1 & 0 & 0 & -x_{2} & -x_{1}
\end{array}\right] ;
$$

moreover, it is set $\rho=1$, so that the plastic multiplier is approximated in the typical subset $\Omega^{j}$ by a uniform function, i.e. $\dot{\gamma}^{j}=\dot{\hat{\gamma}}^{j}$.

Taking into account the equations (9) and (10), the flow rule equation (2) in the subset $\Omega^{j}$ takes the form:

$$
\dot{\boldsymbol{\pi}}^{j}=\left(\mathbf{r}^{j}\right)^{T} \dot{\widehat{\gamma}}^{j} \hat{\mathbf{N}}\left(\widehat{\boldsymbol{\sigma}}^{j}\right) \quad \text { with } \quad \hat{\mathbf{N}}\left(\widehat{\boldsymbol{\sigma}}^{j}\right)=\mathbf{N}\left(\mathbf{P}^{j} \widehat{\boldsymbol{\sigma}}^{j}\right)
$$

From Equation (12) it can be highlighted that the representation form for the inelastic strain rate depends on the approximations introduced for the stress and plastic multiplier.

Substituting the introduced approximations in equations (6) and (12) for each subset $\Omega^{j}$ the discretized form of the yield function and of the evolution law are recovered. Finally, the evolution problem is solved adopting a step-by-step backward Euler algorithm. At each time step, the solution of the evolution problem is performed by establishing a predictor-corrector strategy. Details on the numerical model can be found in [7].

\section{NUMERICAL TEST}

In this section, the performance of the proposed homogenization procedure is verified on the repetitive UC representative of a realistic application, subjected to periodic boundary conditions. The microstructure of the $\mathrm{UC}$ is characterized by a plastic matrix embedding elastic circular inclusions with volume fraction equal to $36 \%$. The UC geometry is shown in Fig. 1a; due to symmetry only one quarter of the UC is modeled. The following geometrical parameters are assumed: $a=0.5 \mathrm{~mm}$ and $r=0.34 \mathrm{~mm}$. The material parameters are collected in Table 1 .

The localization matrices $\mathbf{L}^{\varepsilon, j}$ and $\mathbf{L}^{\pi^{i}, j}$ are evaluated performing linear elastic finite element analyses of the UC subjected to each component, assumed equal to one, of $\bar{\varepsilon}$ and of $\pi^{i}$, respectively, determining the perturbation strain at each Gauss point of the finite element mesh. The MxTFA homogenization analysis is performed discretizing the UC with 7 subsets (6 subsets for the matrix and 1 subset for the inclusion), as shown Fig. 1b. Thus, the number of linear elastic pre-analyses is equal to the 3 components of $\bar{\varepsilon}$ plus the number of the subsets times the 7 components of $\widehat{\sigma}^{j}(3+6 \times 7=45)$; the number of internal variables results $6 \times 7=42$. For the finite element model employed to perform the linear elastic pre-analyses and to compute the micromechanical reference solution, the quarter of UC is discretized using $2 \mathrm{D}$ plane stress four-node finite elements. In particular, 304 finite elements are used, 176 for the matrix and 128 for the inclusion. The internal variables of the nonlinear micromechanical analysis are equal to $176 \times 4 \times 3=2112$, significantly higher than the ones of the MxTFA. 
The results obtained with the homogenization procedure are compared with nonlinear micromechanical analyses (FEA), carried out using finite elements characterized by elastic and plastic constitutive models.

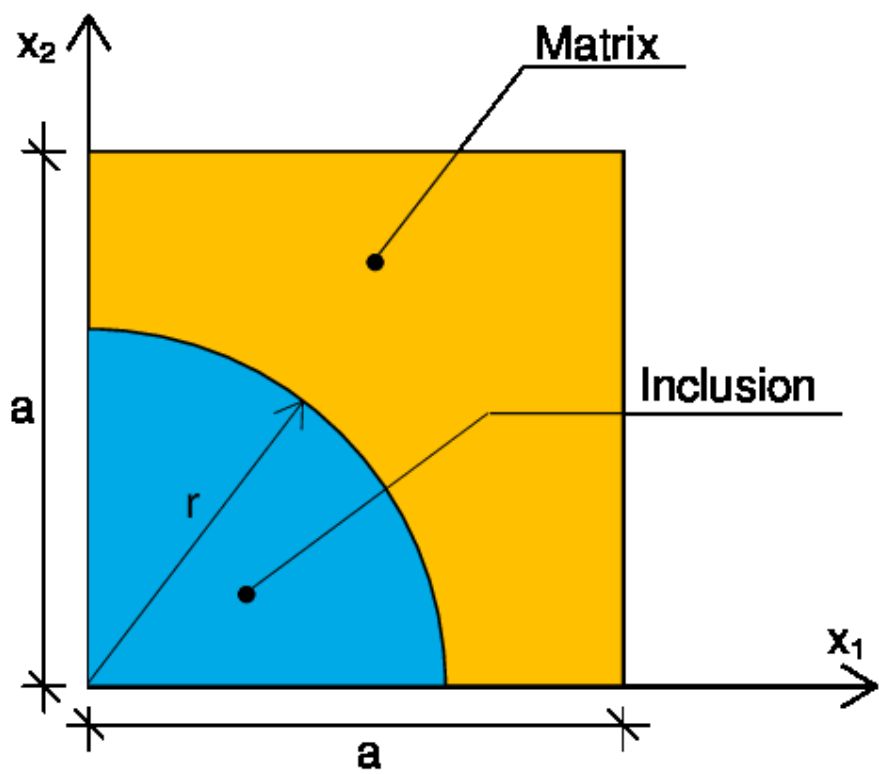

(a)

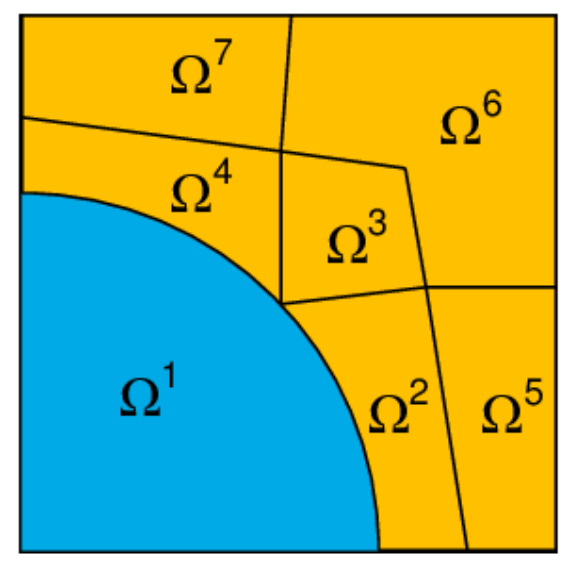

(b)

Figure 1: Geometry of the UC (a) and subset discretization (b).

\begin{tabular}{ccccc}
\hline Constituent & $E[\mathrm{MPa}]$ & $\nu$ & $\sigma_{y}[\mathrm{MPa}]$ & $H[\mathrm{MPa}]$ \\
\hline Inclusion (elastic material) & 410000 & 0.19 & - & - \\
Matrix (plastic material) & 75000 & 0.33 & 426 & 2894 \\
\hline
\end{tabular}

Table 1: Material properties of the constituents.

In this test the UC is subjected to uniaxial loading along the $x_{1}$-axis, with the average strain $\bar{\varepsilon}_{11}$ monotonically increased until the final value $\bar{\varepsilon}_{11}=0.03$. Fig. 2 shows the constitutive response in both cases of elasto perfectly plastic and linear hardening (LH) of the equivalent homogenized material in terms of $\bar{\sigma}_{11}$ and $\bar{\varepsilon}_{11}$, together with the micromechanical reference solution. As it can be noted, the MxTFA is able to accurately reproduce the overall behavior of the UC. Moreover, the final value of the average stress $\bar{\sigma}_{11}$ obtained with the MxTFA is compared with the reference one deriving from the micromechanical analysis in Table 2; as it can be noted, the error is less than $12 \%$.

\section{CONCLUSIONS}

A new mixed nonuniform TFA for the analysis of periodic composites characterized by linear isotropic hardening is proposed. The inelastic field is recovered from the equilibrated stresses and plastic multiplier parameters, that are the independent variables of the problem. The evolution equations involving the internal variables are rationally derived from a variational principle based on the complementary formulation. 


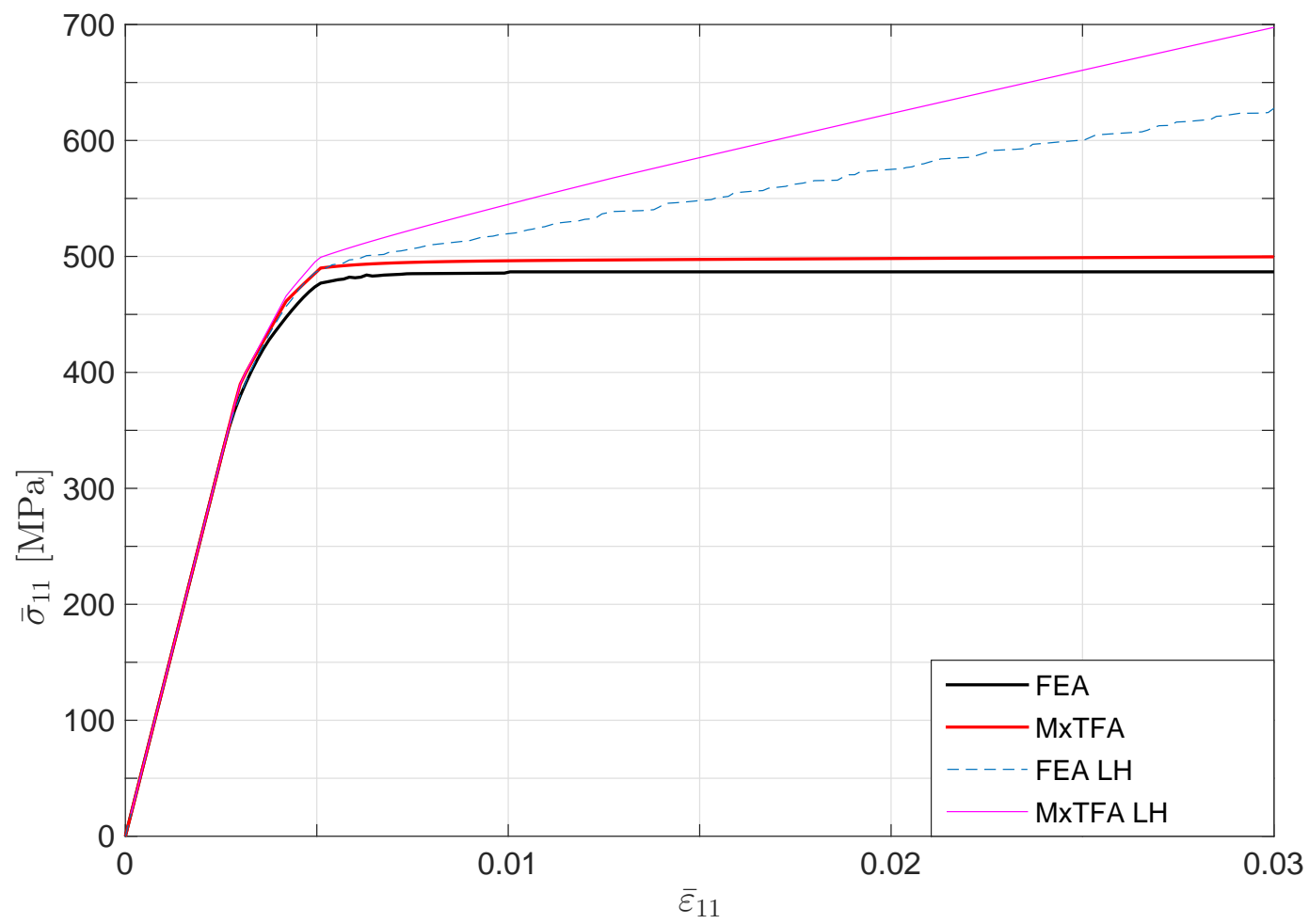

Figure 2: Mechanical response of the UC subjected to uniaxial loading.

\begin{tabular}{lcc}
\hline Type of analysis & $\bar{\sigma}_{11}(\mathrm{MPa})$ & Error $(\%)$ \\
\hline FEA & 486.71 & - \\
MxTFA & 499.66 & 2.66 \\
FEA LH & 627.73 & - \\
MxTFA LH & 697.53 & 11.11 \\
\hline
\end{tabular}

Table 2: Uniaxial loading: final value of the average stress.

The numerical example shows the ability of the proposed MxTFA technique in reproducing the micromechanical nonlinear response for periodic composite characterized by Mises linear isotropic hardening behavior.

The presented approach is much less expensive than the micromechanical finite element analyses from a computational point of view. In fact, in the proposed approach the elasto-plastic problem is solved at each time-step iteration at subset level, adopting a variational formulation resulting in a system of equation involving a number of internal variables significantly lower than in the nonlinear micromechanical analysis.

Because of the computation efficiency, the proposed MxTFA approach results very promising for being implemented at the Gauss point level for the development of an effective multiscale procedure.

Acknowledgments The financial supports of PRIN 2010-11 project "Advanced mechanical modeling of new materials and technologies for the solution of 2020 European challenges" 
CUP n. F11J12000210001 and of the University of Cassino and of the Southern Lazio are gratefully acknowledged.

\section{REFERENCES}

[1] G. Dvorak, Transformation field analysis of inelastic composite materials, Proceedings of the Royal Society of London A 437 (1992) 311-327.

[2] J. Michel, P. Suquet, Nonuniform transformation field analysis, International Journal of Solids and Structures 40 (2003) 6937-6955.

[3] S. Marfia, E. Sacco, Micromechanics and homogenization of sma-wire-reinforced materials, Journal of Applied Mechanics 72 (2005) 259-268.

[4] F. Fritzen, T. Böhlke, Three-dimensional finite element implementation of the nonuniform transformation field analysis, International Journal for Numerical Methods in Engineering 278 (2010) 186-217.

[5] S. Marfia, E. Sacco, Multiscale damage contact-friction model for periodic masonry walls, Computer Methods in Applied Mechanics and Engineering 205-208 (2012) 189-203.

[6] V. Sepe, S. Marfia, E. Sacco, A nonuniform TFA homogenization technique based on piecewise interpolation functions of the inelastic field, International Journal of Solids and Structures 50 (2013) 725-742.

[7] F. Covezzi, S. de Miranda, S. Marfia, E. Sacco, Complementary formulation of the tfa for the elasto-plastic analysis of composites, Composite Structures 141 (2016) In press.

[8] J. Simo, J. Kennedy, R. L. Taylor, Complementary mixed finite element formulations for elastoplasticity, Computer Methods in Applied Mechanics and Engineering 74 (1989) 177-206.

[9] A. Bilotta, L. Leonetti, G. Garcea, Three field finite elements for the elastoplastic analysis of 2d continua, Finite Elements in Analysis and Design 47 (2011) 1119-1130.

[10] F. Daghia, S. de Miranda, F. Ubertini, Patch based recovery in finite element elastoplastic analysis, Computational Mechanics 52 (2013) 827-836.

[11] G. Castellazzi, S. de Miranda, F. Ubertini, Adaptivity based on the recovery by compatibility in patches, Finite Elements in Analysis and Design 46 (2010) 379-390.

[12] G. Castellazzi, S. de Miranda, F. Ubertini, Patch based stress recovery for plate structures, Computational Mechanics 47 (2011) 379-394. 